\title{
Phänomen Neurobiologie der Sprache
}

\author{
Hans Schachl*
}

\begin{abstract}
From phylogeny to ontogeny arises the complex phenomenon of language. This paper aims to illustrate this complexity of language by describing the functions and areas of the brain responsible for listening, speaking, and reading, followed by an exploration of the interaction of emotion and cognition. Furthermore, the paper briefly addresses the special fascination of children's language development as well as the relationship between music and language and the positive effects of music on language development. Additionally, the paper touches upon the topic of "reading" in an application-oriented manner.
\end{abstract}

Key words: language, evolution, neurobiology, understanding and speaking, reading, memory, development

\footnotetext{
* Private Pädagogische Hochschule der Diözese Linz hans.schachl@ph-linz.at
} 


\section{Die Evolution der Sprache}

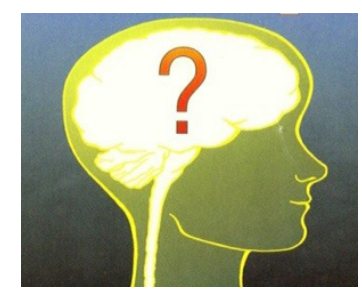

Abbildung 1: Schachl 2012, Titelblatt

Das Streben nach Erkenntnis trieb und treibt uns voran. Das gilt natürlich auch für das Phänomen Sprache. In der Hirnforschung müssen wir insgesamt aber bescheiden sein: „Wenn das Gehirn des Menschen so einfach wäre, dass wir es verstehen könnten, dann wären wir so dumm, dass wir es doch nicht verstehen würden“. (Gaarder 1996, 392)

Am Anfang der Sprachentwicklung stand und steht die Kommunikation, und zwar sowohl in der evolutionären als auch in der individuellen Entwicklung.

„Im Anfang war die Tat“ schreibt Goethe im Faust (Goethe 1808, 80-81). Das bedeutet, dass der Druck zur Erfindung der Kommunikation wahrscheinlich aus der Notwendigkeit, sich über die „Tat“ (die Jagd, die Planung des täglichen Lebens etc.) auszutauschen erfolgte! Einen guten Einblick in die Evolution der Sprache, besonders auch bezüglich der Unterschiede zwischen der Kommunikationsfähigkeit von Primaten und Menschen, und auch in die kindliche Entwicklung gibt das Video „Language and the Human Brain“ von Elman (2008). Siehe auch das Video: CARTA „How Language Evolves: Language in the Brain“.

Menschen mit eingeschränkter sprachlicher Kommunikation kann durch die sogenannte „unterstützte Kommunikation“ geholfen werden. Dabei wird auf „ursprüngliche“ Formen zurückgegriffen: Mimik, Gestik, Blickrichtung, Gebärden, aber auch technische Hilfsmittel, wie Symbole für Buchstaben in einer Tablet-Kommunikations-App, kommen erfolgreich zum Einsatz (Hobel 2017).

Die Entwicklung der menschlichen Sprache bedurfte einer Weiterentwicklung der schon bei den Primaten vorhandenen Sprachzentren, der Umgestaltung des Kehlkopfes und vor allem einer Vergrößerung des Frontalhirns, sowie auch einer Vermehrung der Nervenzellen mit komplexer Vernetzung. Aber die Areale für das Sprechen, Zuhören und Verstehen, oder gar für das Lesen, sind nicht von Anfang spezialisiert für die Sprache entwickelt worden. Die Sprachnetzwerke entwickelten sich aus den evolutionär angelegten Grundfunktionen der Wahrnehmung, die durch die sprachspezifischen Inputs genützt und modifiziert werden (Schlaggar und McCandliss 2007, 494). Die wesentlichen Prozesse finden in den sensiblen Phasen statt und führen zur Komplexität 
der Sprache. Unter „Sensiblen Phasen“ versteht man Zeiträume in der Hirnentwicklung, in denen die Nervennetze besonders leicht durch Erfahrung ausgebaut und auch verändert werden können. Die Sensiblen Phasen sind für unterschiedliche Funktionen unterschiedlich lang: Im sogenannten „Drei-Phasen-Modell“ (Bardin 2013, 55) wird die Entwicklung der Lernfähigkeit des Gehirns in „überlappenden Wellen“ beschrieben: Die Areale für die Sinne öffnen sich im Säuglingsalter und schließen sich gegen Ende der frühen Kindheit (Vorschulzeit, erste Grundschulzeit); die Sensiblen Phasen für die Sprache und andere höhere Leistungen beginnen später und dauern auch länger. Eine gewisse Plastizität bleibt das ganze Leben lang, wird jedoch mit zunehmendem Alter deutlich schwächer.

\section{Die Komplexität der Sprache}

Dazu zunächst ein ganz schneller Überblick:

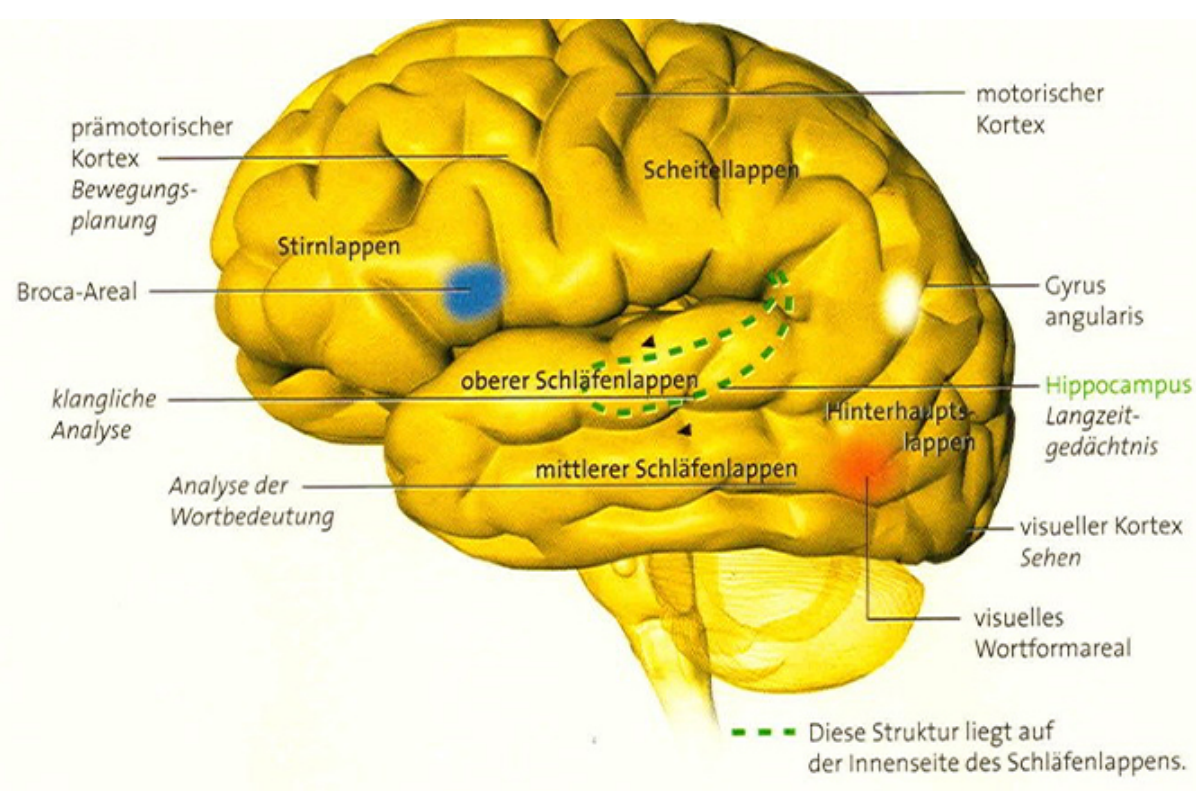

Abbildung 2: Reiter 2014, 64

Zum Auswählen, Vergleichen, Bewerten, für die Grammatik, das Sinnerfassende Hören und Lesen sind folgende visuelle und auditorische Areale notwendig: das visuelle Wortform-Areal; die Schläfenlappen-Areale; das Broca-Areal und das Frontalhirn. Das Frontalhirn kooperiert mit dem Langzeitgedächtnis im Cortex (Wortschatz, Semantik). Wichtig ist auch das Kleinhirn. Hippocampus und Präfrontaler Cortex sind wichtige Arbeitsspeicher.

Das Limbische System (in der ,Tiefe des Gehirns) steuert die Emotionen bei. Auch das Aufmerksamkeitssystem ist involviert. Für das Lesen und Sprechen ist auch die Mo- 
torik der Augenbewegungen und der Gesichts- und Halsmuskulatur notwendig. Der Gyrus angularis ist als Assoziationsareal von Bedeutung. Nun etwas detaillierter:

Nun etwas detaillierter:

\section{Die Vorgänge beim Zuhören und Sprechen}

Das „linguistic decoding“ (Gehirn\&Geist SPEZIAL 2011, 68; Max-Planck-Gesellschaft 2015b, n.p.) erfordert beim Zuhören: die Aufnahme der Schallwellen im Auditorischen Cortex (im oberen Schläfenlappen) und das Entschlüsseln der Phoneme und Silben. Schon dieser erste Analyse-Prozess der grundlegenden „features“ (Chang 2015) ist komplex. Für diese ersten Stufen der Sprach-Segmentation werden „neurale Oszillationen“ (Delta/Theta/Gamma-Wellen) im Auditorischen Cortex angenommen (Giraud 2017). In der weiteren Verarbeitung kommt es zur Einbeziehung anderer Hirnregionen zur Entschlüsselung von Bedeutung, Emotionen, Tonfall, Rhythmus: In der Amygdala (als Teil des Limbischen Systems) werden die Emotionen erzeugt und die eigene emotionale Reaktion eingeleitet. 250-350 ms nach dem Sprechbeginn werden Struktur und Bedeutung der Worte entschlüsselt, und zwar zunächst im Wernicke-Areal in der linken Hirnhälfte; dann extrahieren der vordere Schläfenlappen und der vordere frontale Cortex in beiden Hirnhälften die Bedeutung der Worte. Um die Bedeutung bewusst zu erfassen, müssen die Wörter mit dem Gedächtnis assoziiert werden. Das passiert in einem Teil des Frontallappens (Arbeitsgedächtnis); das Gedächtnis selbst ist allerdings in vielen Teilen des Gehirns zu finden.

Ähnliche Prozesse gibt es beim Sprechen: Das Verstehen gesprochener Wörter erfordert einen kontinuierlichen Prozess der Aktivierung lexikalischer und semantischer Funktionen, bis es zur eindeutigen Erkennung eines Wortes kommt. 


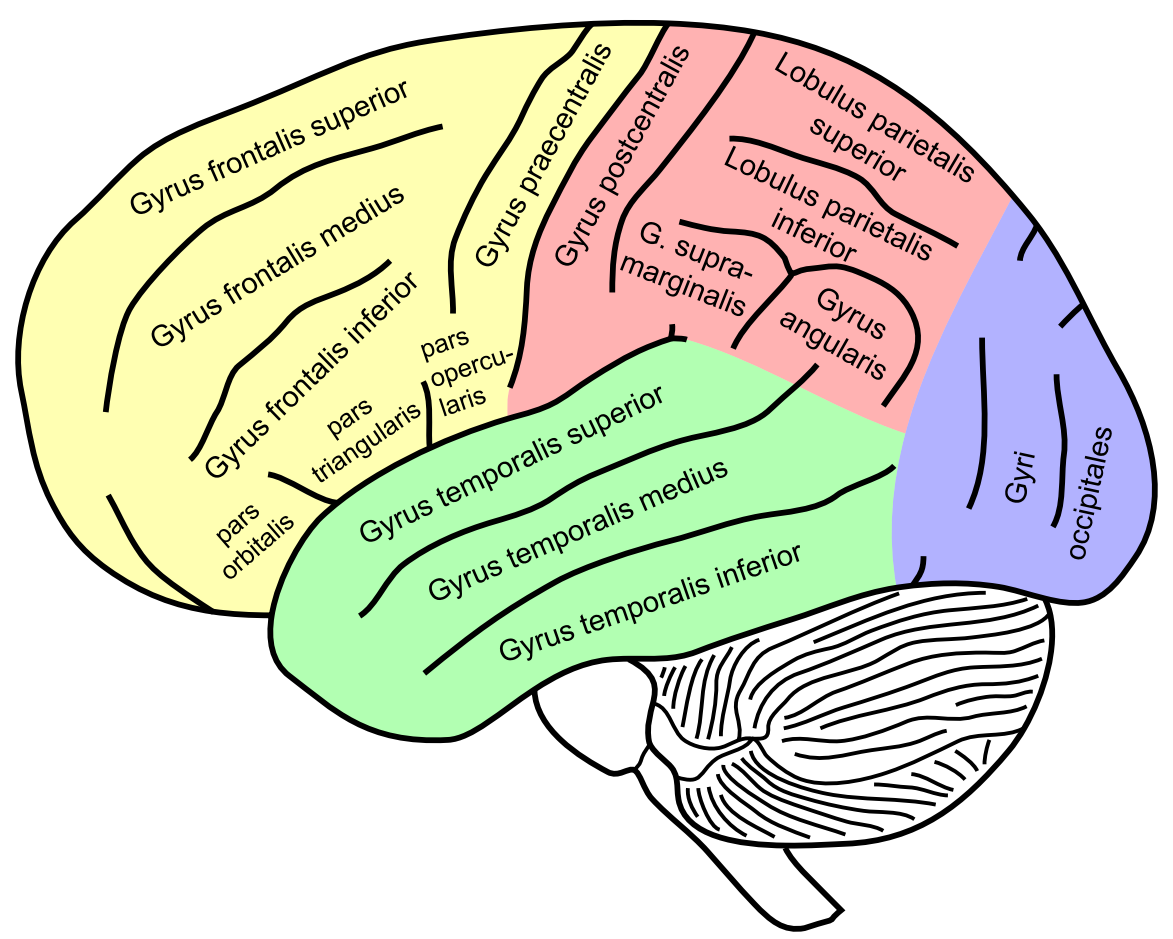

Abbildung 3: https://ca.wikipedia.org/wiki/Circumvoluci\%C3\%B3_frontal_inferior

Dieser komplexe Prozess bis zum bewussten Erkennen dauert 400 bis $500 \mathrm{~ms}$ und umfasst Bereiche vor allem im Gyrus temporalis und im Gyrus frontalis sowie im Gyrus angularis (Kocagoncu et al. 2017). Der linke Gyrus temporal superior hat auch eine spezielle Rolle beim Lesen durch die Integration orthographischer und phonologischer Prozesse (Schlaggar und McCandliss 2007, 480), der Gyrus temporalis medius ist gemeinsam mit den Frontalhirnregionen involviert in die Erkennung der Semantik (Fedorenko 2015).

Die Regionen um die Sylvische Furche (zwischen Schläfen- und Stirnlappen) herum (Perisylvischer Cortex) ist auf dem Weg zum Frontallappen (linker Gyrus frontalis inferior) für das „tuning“ von „low level speech features“ (voice onset time, Phoneme, Silben, Wortgrenzen) zuständig (Berezutskaya et al. 2017). Für die Konstruktion einer hierarchischen linguistischen Struktur (Wörter, Phrasen, Sätze) müssen hierarchische neurale Prozesse stattfinden, die „grammar-based“ gesteuert werden (Ding et al. 2016; Heer et al. 2017). 


\section{Das Visuelle Wortform-Areal (VWFA)}

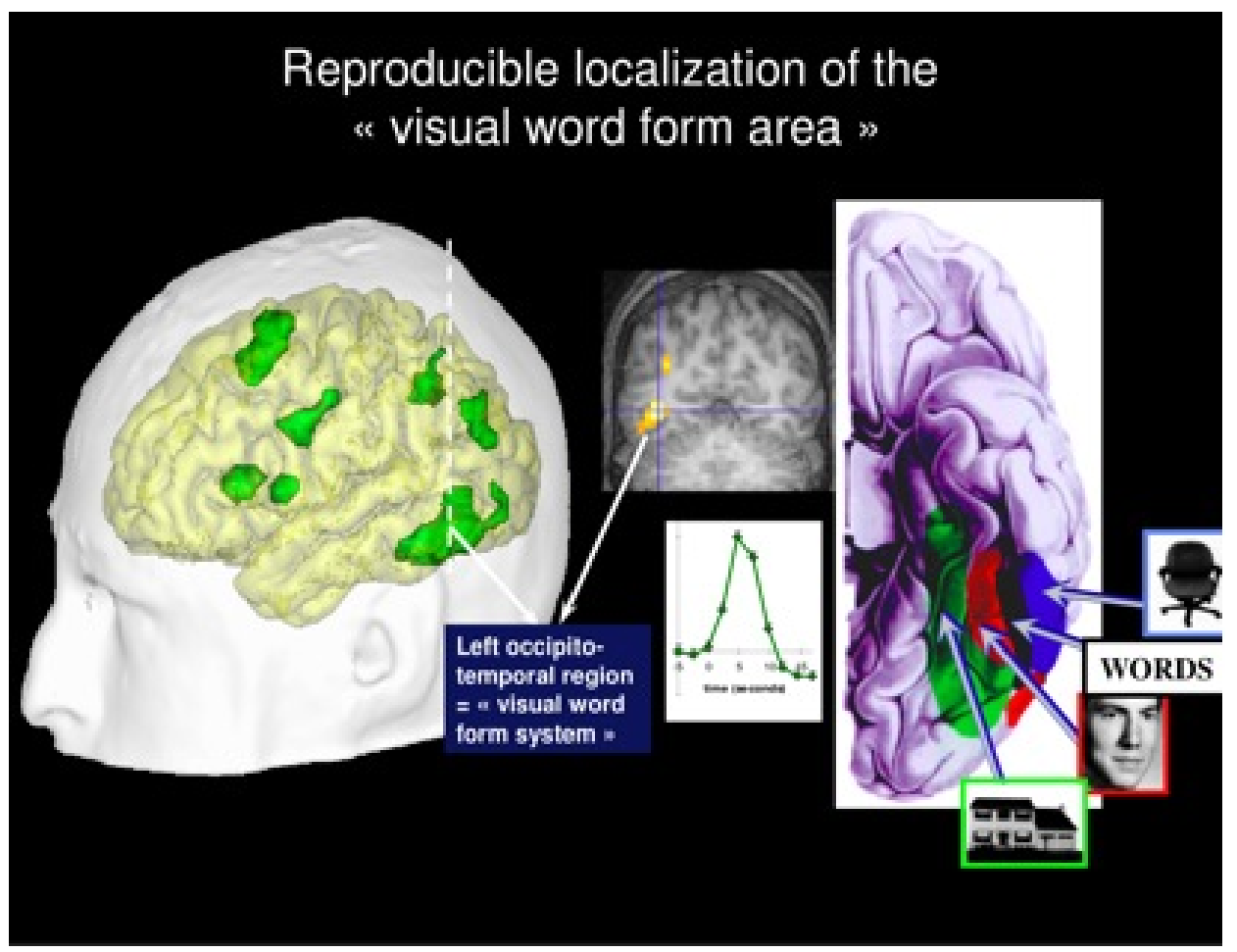

Abbildung 4: Dehaene 2008, http://www.dyslexia-international.org/neuroscience

Dieses Hirnareal befindet sich gleich in der Nähe des primären visuellen Zentrums. Das VWFA wird beim Lesen aktiviert. Es identifiziert die Form von Buchstabenketten; es ist ganz auf geschriebene Wörter spezialisiert, bei reinen Linien-Zeichnungen oder gesprochenen Wörtern bleibt es stumm (Wolf C. 2010, 16; Dehaene und Cohen 2011).

Das VWFA ist umgeben von den Feldern für Objekte und Gesichter. Eine derart ,junge' Funktion wie das Lesen (Erfindung der Schrift vor 5000 Jahren) kann nicht evolutionär angelegt sein. Auf der Basis dieser Überlegung formulierten Dehaene und Cohen (2011) die „neuronal recycling“-Hypothese: „Reading acquisition partially recycles a cortical territory evolved for object and face recognition, the prior properties of which influenced the form of writing systems" (254).

Die Evolution dürfte also die visuelle Basis für die Schriftentwicklung bereitgestellt haben, als ganz allgemein zuständig für das Erkennen von Objekten und Formen („preexisting cortical systems" for "extracting configurations of object contours" nach Dehaene und Cohen 2011, 254). Dazu passt auch, dass das VWFA bei allen Menschen an der gleichen Stelle ist, ganz gleich, ober wir Englisch, Chinesisch, Deutsch, etc. lesen (Dehaene und Cohen 2011, 256). Eine gute Erklärung der Hirnprozesse beim Lesen gibt Dehaene (2013) im Video „Reading the Brain“. 
Diese „Neuroplastizität“ (Veränderbarkeit) durch Aktivität zeigt sich ganz besonders darin, dass das VWFA auch von anderen Sinnesreizen benützt werden kann: Das Lesen blinder Menschen mit dem Braille-System (also über taktile Reize) aktiviert das VWFA ebenfalls, das heißt, „it may possess a general capacity for identifying shapes“ (Dehaene und Cohen 2011, 260). Ein ganz interessanter Befund ist allerdings: Bei von Geburt an blinden Personen zeigte das VWFA Reaktionen auf mit Braille geschriebene Wörter und auch auf die grammatikalische Verarbeitung gesprochener Sprache; ersteres natürlich auch bei Sehenden, aber zweiteres nicht (Kim et al. 2017).

Beeindruckend ist die Neuroplastizität bei Kindern, die durch eine prä- oder perinatale Schädigung von Geburt an taub sind. Ihr Auditorischer Cortex wird nicht durch auditorische Elemente erregt, sondern durch visuelle. Siehe dazu auch das Video von Stiles (2008) „Brain Development“ und auch das Video von Elman (2008) „Language and the Human Brain“ ab Minute 26:00.

Das VWFA entwickelt sich funktionell für das Lesen durch das Lesen: Die Aktivität des VWFA korreliert linear mit der Lese-Performance (Dehaene und Cohen 2011, 258; Saygin et al. 2016; Schlaggar und McCandliss 2007, 480). Diese Spezifizität für das Lesen zeigt sich zum Beispiel auch durch eine besondere elektrische Aktivität, ein ERP (EventRelated Potential), und zwar durch die „N170“-Welle, die 170 ms nach der Präsentation des Lesereizes im linken „ventral occipito-temporalen“ Bereich (inklusive VWFA) ausgelöst wird. Diese lese-spezifische Welle muss sich aber erst vom Erstlesen zum Erwachsenen entwickeln (Schlaggar und McCandliss 2007, 485). Das VWFA ist nicht nur „bottom up“ für die Encodierung von Buchstabenketten tätig, sondern wird auch „top down" (Aufmerksamkeit, Semantik, Gedächtnis, Gefühle) beeinflusst (Schlaggar und McCandliss 2007, 480). Dieses Zusammenspiel von ,bottom up` und ,top down' ist ein allgemeines Prinzip der Wahrnehmung. 


\section{Kognition und Emotion}

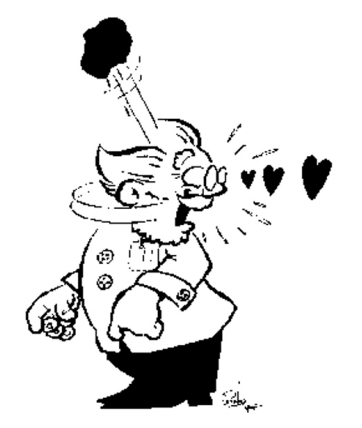

Abbildung 5: Schachl (2012, 19)

Eine Trennung von ratio und emotio, von Verstand und Gefühl, ist hirnbiologisch nicht nachvollziehbar. Es wohnen nicht „zwei Seelen in meiner Brust“, wie es in Goethes Faust $(1808,73)$ heißt (siehe Cartoon, Abbildung 5). „Gefühle haben einen starken Einfluss auf Lern- und Gedächtnisleistungen" (Roth 2011, 180). Die Art der Wechselwirkung zwischen Gefühlen und Verstand ist ein wesentliches Merkmal der Persönlichkeit des Menschen (Roth 2011, 42).

Wie wirken Verstand und Gefühle zusammen?

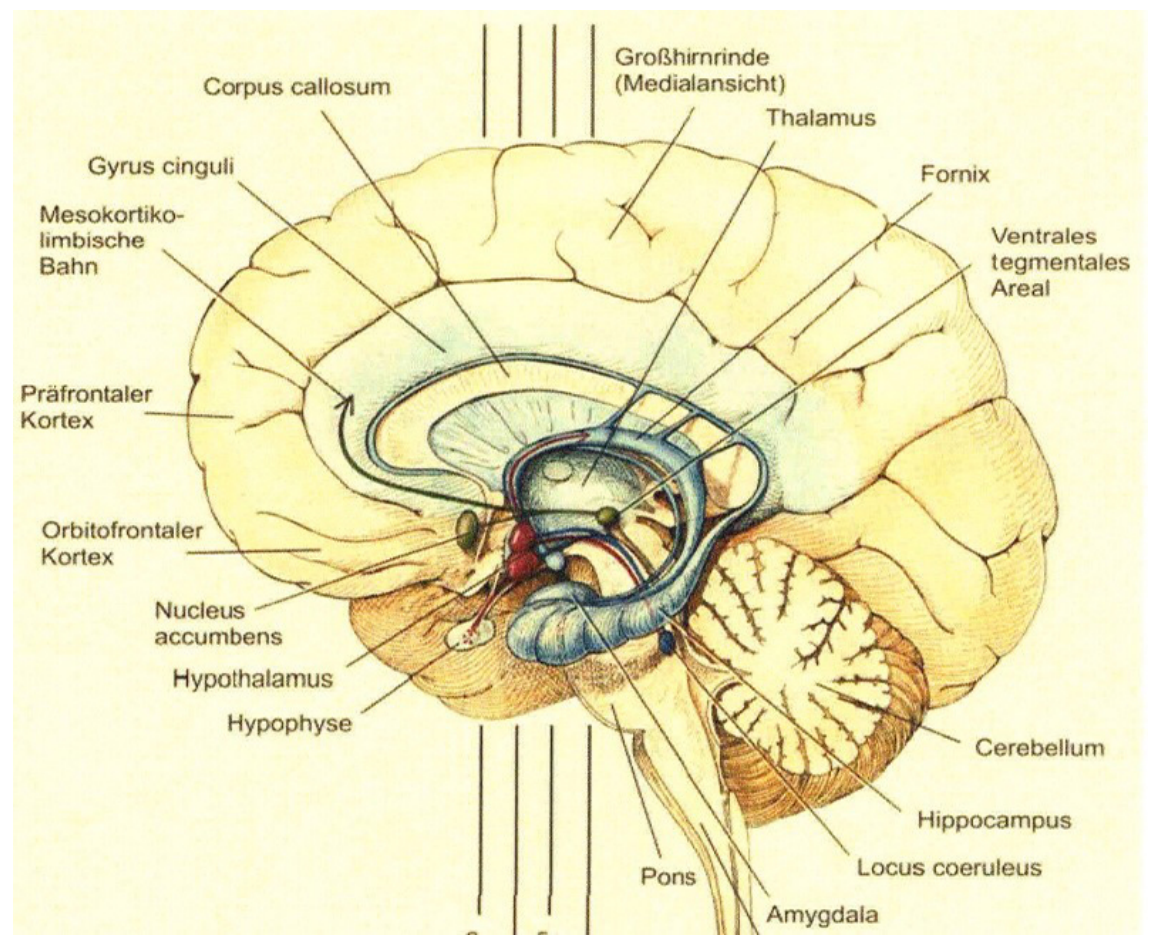

Abbildung 6: Förstl, Hautzinger, Roth 2006, 5 
Das Zusammenwirken von Gefühl und Verstand kann hier nicht detailliert beschrieben werden, daher wird auf die zusammenfassende Theorie über „den Sitz und die Physiologie der Seele“ (besser: der Psyche) von Roth und Strüber (2015) verwiesen: Die Netzwerke für die Psyche werden als Limbisches System zusammengefasst. Roth-Strüber (2015, 63-64) unterscheiden drei „limbische Ebenen“ (eine untere, mittlere, obere), der noch eine „kognitiv-sprachliche“ Ebene gegenübersteht. Sie werden als die „Vier Ebenen der Persönlichkeit“ bezeichnet. Die Aktivitäten (vegetative Körperfunktionen, Emotionen) der unteren (mit Hypothalamus, Amygdala, u. a.) und mittleren (mit Basolateraler Amygdala, Nucleus accumbens, Ventral Tegmentalem Areal, Thalamus, Hippocampus u. a.) limbischen Ebene sind unbewusst. Auf bewusster Ebene bildet die obere limbische Ebene das „individuelle-soziale Ich“ und die kognitive Ebene das „kognitivkommunikative Ich“. Die obere limbische Ebene ist für „Verhaltensplanung“ mit dem Ziel der „Bewertung“, ob die geplanten Handlungen positive oder negative Konsequenzen haben werden, zuständig. Hier finden sich wichtige Integrationszentren zwischen Basis-Emotionen und Kognition. Bei Schädigungen im Orbitofrontalen und Präfrontalen Cortex kommt es zum Verlust der emotionalen Kontrolle und damit des angemessenen Sozialverhaltens.

Die „kognitiv-sprachliche“ Ebene mit dem Dorsolaterlaen Präfrontalen Cortex, dem Ventrolateralen Präfrontalen Cortex, dem Broca-Sprachzentrum, u. a. (Roth-Strüber, 2015, 87-91) hat als Aufgaben die Planung und Vorbereitung von Handlungen, das Problemlösen, Erinnern, Vorstellen, Denken, die Koordination, etc. Zusammenfassend lässt sich folgendes sagen: Die Areale der Kognition (Hippocampus, Frontal Cortex, etc.) werden stark beeinflusst von den Arealen der Emotion (Limbisches System mit Amygdala, Belohnungssystem mit Nucleus accumbens und Ventral Tegmentalem Areal, Hypothalamus, etc.).

Das hat für Lehren und Lernen eine ganz wesentliche Konsequenz: Gefühle sind nicht nur subjektive Erfahrungen, sondern auch für kognitive Leistungen notwendig.

\section{Kurz- und Langzeitgedächtnis}

Das Frontalhirn muss bei der Sprache vieles tun: Auswählen hinsichtlich Bedeutung, Vergleichen, Bewerten, eben ,Nachdenken', Philosophieren, etc.! Dieser Teil des Gehirns ist maßgeblich dafür verantwortlich, dass aus Buchstaben, Lauten, etc. Gedanken werden. Da bei der Wahrnehmung von Sprache (Hören, Lesen) ja nicht nur einzelne Wörter erkannt werden müssen, sondern ganze Sätze, ist das ,Arbeitsgedächtnis` notwendig: Es müssen die einzelnen Einheiten präsent gehalten und mit dem Langzeitgedächtnis abgeglichen werden, um den Zusammenhang und Sinn zu begreifen. Für diese Arbeitsspeicherfunktionen sind vor allem der Hippocampus und der Präfrontale Cortex zuständig. 
Wichtig: Ein vertrautes, häufiges Wort wird schon nach 300-400 ms erkannt. Je mehr solche Wörter ein Text enthält, desto leichter begreifen wir ihn. ,Sinnerfassendes Lesen“ muss also vom ,Bekannten zum Neuen' gelernt werden! Verschiedene Wortarten werden auch unterschiedlich gespeichert und sind daher unterschiedlich lesbar: So werden Verben besser aufgenommen, weil sie auch Hirnareale ansprechen, die für Bewegungen zuständig sind. Konkrete Begriffe werden besser gelesen, weil sie Erlebnisse (Autobiographisches Gedächtnis) und Emotionen (Limbisches System) stärker einbeziehen. Daher gilt allgemein für das Lernen und damit auch für das Lesen: Vom Konkreten zu Abstrakten.

\section{Die Rolle des Kleinhirns}

Das Kleinhirn ist an vielen Funktionen beteiligt: Es ist - neben der Kontrolle von Muskelbewegungen und des Gleichgewichts - auch ein Gedächtnisbereich vor allem für implizit ablaufende Motorik- Automatismen (,Prozedurales Gedächtnis‘, z. B. auch für die Schreibbewegungen), und es ist in Emotionen und eben auch in Sprachfunktionen involviert.
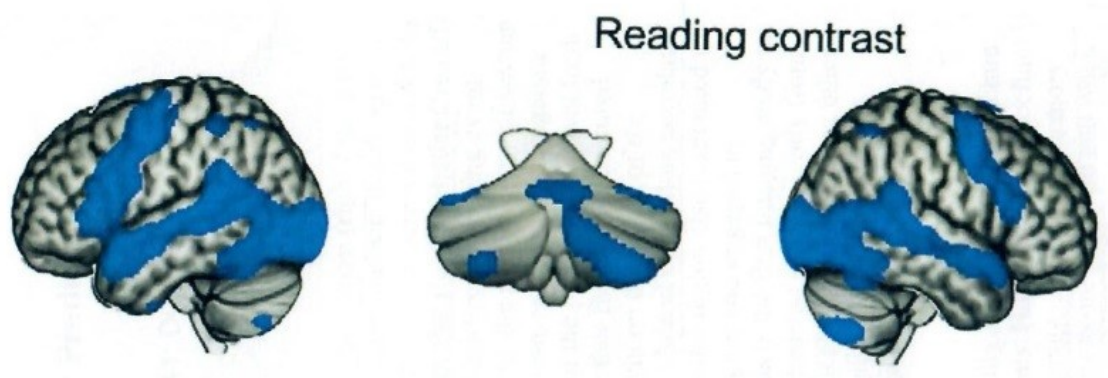

Abbildung 7: Lesage, Hansen, Miall 2017, Fig. 4

Abbildung 7 zeigt die Aktivitäten beim Lesen, bei der Ver- bzw. Bearbeitung des Satzes („reading contrast“) in verschiedenen Hirnbereichen, auch im Kleinhirn: Im Prozess des Entstehens eines Satzes werden die nächsten Wörter, vorhergesagt ${ }^{\star}$.

Das Areal im rechten Kleinhirn ist bei diesem Vorhersage-Prozess aktiv, und zwar aktiver bei einem unerwarteten Wort (prediction error) als bei einem erwarteten (Lesage, Hansen, Miall 2017). Das Kleinhirn scheint eine Art ,phonologic store‘ zu sein. Allerdings ist für eine Vorhersage eine Involvierung in die Semantik naheliegend. Das heißt, eine Art ,common code' könnte in der phonologischen Bearbeitung wirksam sein. Dafür sprechen andere Befunde, die dem Kleinhirn auch semantische Aufgaben zuweisen (Lesage, Hansen, Miall 2017). Dazu gibt es interessante Befunde zu den involvierten 
neuralen Vorgängen: Schon vor der Präsentation eines Wortes werden die kontextabhängigen ,semantischen Vorhersagen, Erwartungen' durch ein spezielles elektrisches Potential angezeigt, das „semantic readiness potential“ (SRP) genannt wird (Grisoni, Miller und Pulvermüller 2017, 4848):

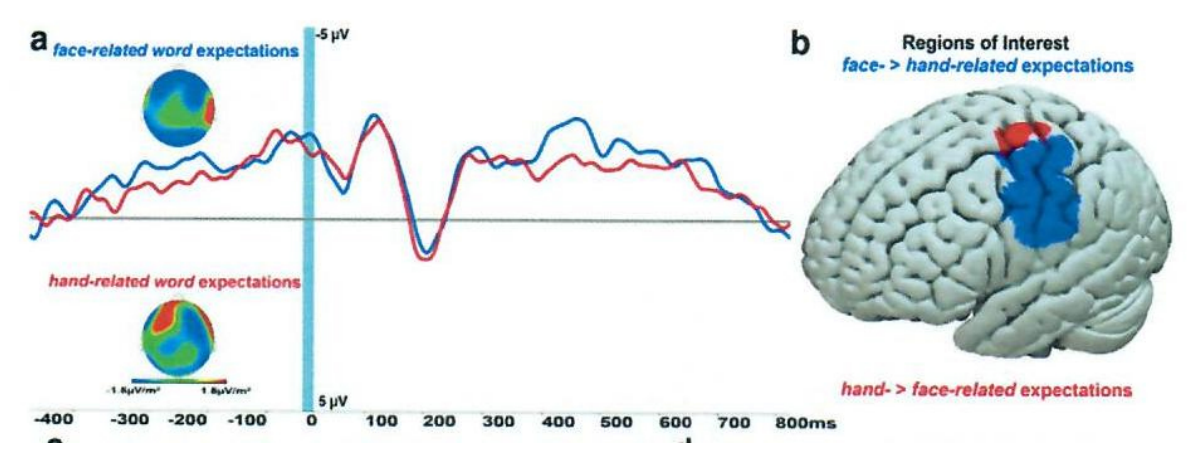

Abbildung 8: Leicht modifiziert nach Grisoni, Miller und Pulvermüller 2017

Diese ,N400-Welle` tritt auf, wenn der vorherige Satz-Kontext zum kommenden semantischen Bereich passt; wenn nicht, dann gibt es kein SRP. Das Potential baut sich ca. $400 \mathrm{~ms}$ vor dem Test-Wort auf (daher $-400 \mathrm{~ms}$ ) und erzeugt dann ein ,Reaktionspotential' unmittelbar nach der Präsentation (siehe Fig. a). Zunächst sieht man diese SRP in den sensomotorischen Arealen (Fig. b), und zwar sehr spezifisch: bei Hand-bezogenen Wörtern (z. B. schreib) oben (in den Handfeldern der Sensomotorik), bei Gesichtsbezogenen Wörtern (z. B. sprich mit mir) darunter (in den Gesichtsfeldern).

\section{Spezialthema „Lesen“1}

Ein kurzes Video zeigt die wesentlichen Bereiche des Gehirns, die beim Lesen kooperieren: „How Does The Reading Brain Work?“ https://www.youtube.com/watch?v=5kB 7GgLIR7M; am 02.09.2014 veröffentlicht. Siehe auch das Video mit Dehaene (2013): Reading the Brain.

Der Weg zum Lesen: Grundlegend für alles Lernen, auch für das Erlernen des Lesens, ist es, die Neugierde und Fantasie kind- und altersgemäß zu ,pflegen'. Dazu gehören unter anderem das Erzählen, das gemeinsame Anschauen von Bilderbüchern, das Vorlesen, etc. Das Lesen beginnt also vor dem Lesen! Das spätere Lesevermögen wird stark davon beeinflusst, wie oft und wie lange die Eltern und andere Bezugspersonen den Kindern vorgelesen haben. Das Vorlesen hilft sowohl bei der Wortschatzerweiterung als auch

1 Siehe dazu auch Kapitel 4 
der syntaktischen und grammatischen Entwicklung. Außerdem lernt das Kind auf dem Schoß, Lesen mit guten Gefühlen der Beziehung zu verbinden. Und auch die Geschichten vermitteln Gefühle und helfen eine ganz wichtige Eigenschaft zu entwickeln, die Empathie. Alle diese Vorstufen werden als „emergente oder frühe Literalität“" (Wolf, M. 2010, 99) bezeichnet. Dies ist verbunden mit dem Verständnis von Bildern und dann auch mit der allgemeinen Sprachentwicklung (Wolf, M. 2010, 102-108). Ebenfalls wichtig ist die Fähigkeit der Bezeichnung von Buchstaben. Kinder erwerben in den meisten Schriftkulturen ein Repertoire an Buchstaben und Wörtern, bevor sie lernen, diese Buchstaben zu schreiben (Wolf, M. 2010, 112). Aus seinen Forschungen über die Hirnprozesse beim Lesen zieht Dehaene für die Leselernmethode folgende Schlüsse (Dehaene in Cook 2010, 24; Dehaene 2013):

Die Ganzwort-Methode sei nicht gehirngerecht, weil das Hirn beim Lernen des Lesens das „serial letter by letter decoding“ braucht. Daher sollten die Kinder mit dem Erlernen von Graphemen (Buchstaben) und Phonemen (grundlegenden Lauten wie z. B. „sch“) beginnen. Bei Erwachsenen findet dieses serial decoding parallel statt; daher ist die Lesegeschwindigkeit größer (Dehaene 2013).

Im Gehirn (im Hinterhaupts- und Schläfenlappen) gibt es zwei Routen des Lesens: eine unten (ventral), die für die parallele Verarbeitung von Buchstaben und Wörtern zuständig ist; und eine zweite oberhalb (dorsal), die eine serielle Verarbeitung Buchstabe für Buchstabe durchführt (Wolf, Barzillai und Norton 2008). Die ventrale Route wird allerdings erst mit zunehmendem Lesen immer besser und schneller (Dehaene 2013), weil sie auf das größere Repertoire zurückgreifen kann. Sie wird dann auch mehr linkshemisphärisch spezialisiert. Die ventrale Route wird als "Bahn des flüssigen Lesens" bezeichnet (Wolf, M. 2010, 168-169). Leseanfänger können die größeren Einheiten hören und segmentieren. Das „Phonembewusstsein“ ist auf jeden Fall für die weiteren Leselernprozesse sehr wichtig. Es kann durch Reime, Lieder, oder Spiele gefördert werden (Wolf, M. 2010, 139-140). Ein Grundproblem ist allerdings: Wenn die Kinder zum schulischen Lesen kommen, haben sie bereits eine sehr unterschiedliche ,Vorgeschichte ihrer Sprachentwicklung. Daraus resultiert ganz wesentlich die Forderung nach ,Individualisierung'. Das bedeutet: Erreichbare Ziele setzen, die unterschiedlichen Potentiale beachten, an den Stärken ansetzen, bei der Korrektur der Schwächen helfen! Dass das methodisch nicht ganz einfach ist, ist klar, aber dennoch führt daran kein Weg vorbei.

Die ,Korrektur der Schwächen“ hat ein sehr wichtiges spezielles ,Anwendungsfeld‘, die Legasthenie/ Dyslexie. Darauf kann hier aus Platzgründen allerdings nicht weiter eingegangen werden. 


\section{Die Vorgänge in den ,Netzen}

Zum wirklichen Verständnis der Funktionen des Gehirns muss man sich auch mit dem ,Innenleben', den elektrochemischen Prozessen in den Nervennetzen, beschäftigen: Geist, Denken, Lernen, Gefühle und natürlich auch Sprache werden von unseren Nervenzellen ,gemacht‘: „Connections really matter“ (Elman 2008).

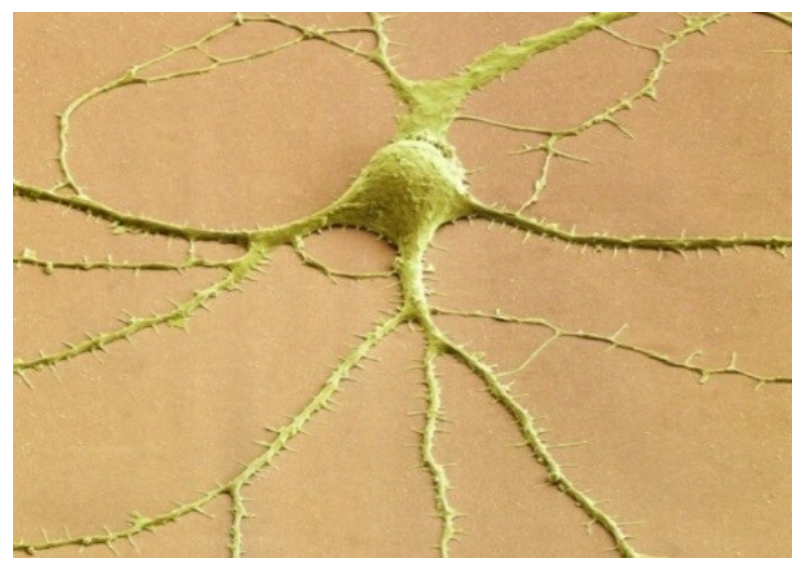

Abbildung 9: Bareither 2014, 43

Abbildung 9 zeigt eine Nervenzelle mit ,Kontaktstellen' (,Spines'). Zur Illustration der Komplexität: Im Gehirn gibt es 86 Milliarden Neuronen (davon etwa $15 \mathrm{Md}$. in der Großhirnrinde, ca. 60 Md. im Kleinhirn), und noch einmal so viele Glia-Zellen. In der Großhirnrinde finden sich bis zu 20.000 Synapsen (Verbindungen) pro Neuron. Dazu kommt die Kommunikation zwischen Glia-Zellen und Neuronen.

Ein Rechenbeispiel: 86 Md. x 10.000 (Durchschnitt) ergeben 860000000000000 (860 Billionen) Synapsen im gesamten Gehirn. Die Synapsen bilden die Grundlage für alle Funktionen, auch für die Sprache. Eine nähere Erläuterung ist hier aus Platzgründen nicht möglich. Eine genaue Darstellung der komplizierten Vorgänge findet sich in Roth und Strüber 2015, 47-54. Das Video „Synaptic Plasticity - How Synapses Spark“ (https://www.youtube.com/watch?v =W9BjRIddG6o) bietet hierzu ebenfalls eine gute Darstellung.

Ein wichtiges Thema für das Verständnis des ,Innenlebens' des Gehirns ist auch die Neurogenese, die Fähigkeit aus Stammzellen neue Nervenzellen zu bilden. ${ }^{2}$

\footnotetext{
2 Siehe dazu z. B. Roth und Strüber 2015, 254; Apple, Fonseca, Kokovay, 2017; Kempermann 2006 und 2017; Kumar et al. 2016; Skaggs 2015; Danielson 2016; Kheirbek/Hen 2015; Bergmann und Huttner 2015; Schoenfeld und Cameron 2015; Berninger und Götz 2009; Shors 2009; Ma et al. 2009; Yao 2016.
} 


\section{Die Entwicklung der Sprache}

Im Kontext der neuronalen Entwicklung ist auch die Entwicklung der Sprache von besonderem Interesse. Diese Entwicklung beginnt bereits im Mutterleib (im letzten Schwangerschaftsdrittel): Die Stimme der Mutter wird am intensivsten wahrgenommen und auch nach der Geburt „erkannt“, und zwar bereits in den ersten Tagen (Berger o. J., 51). Bereits bei zwei Tage alten Babys sind die relevanten Sprachregionen in beiden Hirnhälften aktiv, vor allem aber der rechte Auditorische Cortex. Aber die funktionellen und strukturalen Verbindungen sind noch unreif. Das heißt, „the brain responds to spoken language already at birth, thereby providing a strong biological basis to acquire language, progressive maturation of intrahemispheric functional connectivity is yet to be established with language exposure as the brain develops" (Perani et al. 2011, 16056). Die Abhängigkeit der Hirnentwicklung von den Umweltreizen ist das Grundprinzip des lernenden Gehirns: Der größte Teil der Hirnentwicklung findet nach der Geburt bis ins Kleinkind- und Vorschulalter statt.

Einen interessanten Einblick in die ersten Phasen der Sprachentwicklung ab der Geburt gibt Kuhl (2011) im Video „The Linguistic Genius of Babies“. Mit sechs Monaten kennen die Babys bereits ihren Namen, lange bevor sie ihn sprechen können. Jedes Ding braucht einen Namen: Daher werden zuerst die Substantive gelernt, später die Verben, Adjektive usw. Dabei spielt die ,Form` der Gegenstände eine wichtige Rolle beim Erwerb des Wortschatzes. In diesem Zusammenhang ist interessant, dass bereits sehr früh (schon bei drei Monate alten Babys) eine Verbindung zwischen den Sprachfeldern der gesprochenen Sprache im Temporallappen und dem Visual Word Form Areal (für Objekterkennung; siehe „Neural Recycling Hypothesis“) besteht (Dehaene und Cohen 2011, 256), also bereits hier eine Grundlegung für das spätere Lesen geschaffen wird. Das Zuhören und Verstehen ist früher vorhanden als das verstehende Sprechen. Das zeigt sich auch in der neuralen Entwicklung der wichtigen Verbindungen (dorsale und ventrale Stränge) der Sprachfelder. Und diese Stränge, beispielsweise zum für das Sprechen wichtigen Broca-Feld, müssen sich bei Babys erst ausbilden. Dabei ist die Myelinisierung (Umwicklung der Nervenfasern mit Isolierschichten) wichtig. Die Nervenleitung zu den motorischen Feldern ist früher myelinisiert; das ermöglicht die ersten Sprachproduktionen des „Lallens“ (Perani et al. 2011). Lallwörter sind in vielen Sprachen gleich oder ähnlich: mama, papa, dada, etc. Entscheidend für den Lernfortschritt sind hier die ,Selbstnachahmung' (das „repetitive Babbeln“, Berger o. J., 52) und die Anregung durch die Erwachsenen: Wiederholen und Nachahmen sind der Schlüssel zum Lernen. Die ersten Wörter (,Protowörter` wie Papa, Mama, ...) werden bald zu sogenannten ,Einwortsätzen', die viele Funktionen erfüllen können: Je nach Tonfall und Gesten sind sie Fragen, Wünsche, Bitten, Befehle, Gefühle, etc. Das gilt auch für die ,Zwei-Wort-Sätze‘, die das ,Zeigen' mit verschiedenen Funktionen verbinden.

Scheinbar gibt es mindestens zwei sensible Phasen für den Spracherwerb: Für die Phoneme liegt dieser Zeitraum in den ersten beiden Lebensjahren. Die zweite Phase für 
das Lernen von Syntax und Grammatik dauert länger; sie nimmt erst nach dem 8. Lebensjahr allmählich ab. Aber es gibt keine sensible Phase für die Semantik, weil ja bis zum Tod neue Wörter und Bedeutungen gelernt werden können (Aamodt, Wang 2012, 79). In diesen sensiblen Phasen sind Förderung und Anregung, auch als Basis für das Lesen, besonders wichtig (siehe oben: Stimulierung der Nervennetze und Synapsen). Die Sprachentwicklung geht von diesen ersten Phasen rasant weiter: Die „Benennung“ von Dingen wird durch die zunehmend mehr werdenden Fragen der Kleinkinder intensiviert. Im sogenannten „ersten Fragealter“ dominieren die „WAS“-Fragen, die von den Bezugspersonen ja meist ganz gut beantwortet werden können. Der Wortschatz wächst rapide an. Das „zweite Fragealter“ ist durch die „WARUM“ (denn)-Fragen gekennzeichnet: Hier wird es manchmal sehr schwierig, Kindern Antworten zu geben, die sie schon verstehen können (Schneider und Lindenberger 2012, 195).

Beispiel Niklas (bereits mit drei Jahren!): „Oma, schau der Herr Mond und die Frau Sonne!“ Nach einer Gedankenpause: „Aber warum scheint denn auch der Herr Mond? Es ist ja nicht Nacht!“ (Quelle: privat).

Zur Entwicklung der Grammatik lässt sich folgendes ausführen: Der Behaviorismus meint, der Spracherwerb erfolge ausschließlich durch Umwelteinflüsse, durch Verstärkung und Nachahmung. Diese Prozesse spielen sicher eine wichtige Rolle, erklären aber bei weitem nicht alles. Der Nativismus von Chomsky sagt, dass speziell der Erwerb der Grammatik viel zu komplex und daher nicht durch reine Nachahmung möglich ist. Er nimmt an, dass Kinder mit einem Spracherwerbsmechanismus (LAD - language acquisition device) geboren werden. Dieses Grundprogramm enthält Regeln, die „universell“ für alle Sprachen gelten (z. B. Subjekt-Prädikat-Unterscheidung); daher wird es auch als „Universalgrammatik“ bezeichnet (Blakemore und Frith 2006, 68-69).

Kritiker meinen, dass aber noch mehr notwendig sei als dieses ,Grund-Set' an Regeln, und postulieren den Interaktionismus, der die angeborenen Programme und umweltbedingten Einflüsse in einer Wechselwirkung sieht. Es wird vor allem die Rolle des sozialen Kontextes betont: So zeigt eine Reihe von Studien, dass Kinder aus Familien mit einem niedrigen Sozialstatus eine schlechtere Sprachentwicklung haben, was auch in den für Sprache zuständigen Hirnarealen nachweisbar ist (Wolf, C. 2010, 14-20). Wahrscheinlich haben diese Prozesse (Behaviorismus, Nativismus, Interaktionismus) verschiedene Gewichtung bei den unterschiedlichen Aspekten bzw. Kompetenzen und Komponenten der Sprache. Insgesamt und bei der Grammatik ganz besonders genügt sicher nicht die einfache Nachahmung, sondern das Kind muss auf der Basis des Sprach-„Angebots“ die abstrakten „Regeln“ ableiten und „konstruieren“ (Weinert und Grimm in Schneider und Lindenberger 2012, 436). Kinder sind beim Konstruieren oft sehr kreativ. So verwenden sie z. B. „Pflanzenmann“ für einen Gärtner (Berk 2005, 322). Sie machen aber auch Fehler, die deutlich zeigen, dass sie die Grammatik eben tatsächlich durch „Konstruktion“ erarbeiten (Berk 2005, 323): Mein Spielzeugauto „brichte“ (statt „ist gebrochen“) oder: „Mause“ statt „Mäuse“. 
Zur Sprachentwicklung im Schulkindalter ist folgendes zu bedenken: Beim Schuleintritt gibt es erhebliche Differenzen im Sprachniveau. Die Sprache entwickelt sich natürlich im Schulkindalter intensiv weiter, auch wenn es nicht so sichtbar (bzw. ,hörbar') ist wie in der frühen Kindheit. Viele neue Wörter werden aus dem Kontext mit dem Lesen erworben. Die Kinder benützen die Sprache reflektierter und analytischer. Im Zusammenhang mit der allgemeinen kognitiven Entwicklung vom konkret-operatorischen zum formalen Stadium ist auch das sukzessive Verständnis abstrakter Begriffe zu beobachten: Kauschke, Nutsch und Schrauf (2012, 2-11) konnten durch Reaktionszeitexperimente nachweisen, „dass sich die Qualität und Geschwindigkeit bei der Verarbeitung abstrakter Wörter in der Altersspanne zwischen acht und zwölf Jahren verändert“" (9): Achtjährige verarbeiteten konkrete Wörter richtiger und schneller als abstrakte. Auch die Zwölfjährigen konnten konkrete Begriffe besser verarbeiten, aber die Reaktionszeiten unterschieden sich nicht mehr in Abhängigkeit vom Konkretheitsgrad. Diese Entwicklung vom ,Konkreten zum Abstrakten“ ist insgesamt eine Maxime für den schulischen Unterricht. Dies bedeutet für die Sprache zweierlei: Einerseits ist eine zu frühe und gehäufte Verwendung von abstrakten Begriffen zu vermeiden, andererseits ist die Abstraktionsfähigkeit der Kinder zu fördern. Dabei ist kritisch zu hinterfragen, welche Abstrakta überhaupt Sinn machen. Durch gute Anleitungen in der Schule kommt es auch zu einer fortschreitenden Lautdifferenzierung und Verbesserung der Artikulation. Eine wichtige Aufgabe der Schule ist es, die ,gehobene Sprache‘ zu schulen. Während des Schulkindalters verbessert sich auch die Grammatik: Die Konstruktionen werden komplexer. Auch die durchschnittliche Satzlänge nimmt ständig zu. Verbesserungen der kommunikativen Seite der Sprache (der Sprachpragmatik) sind ebenfalls zu verzeichnen. Es entwickeln sich bessere Gesprächsstrategien, z. B., um zu erreichen, was sie wollen. Die Kinder können auch besser auf Unterschiede zwischen dem, was Menschen sagen und dem, was sie meinen, reagieren. Abschließend ist zu konstatieren, dass es große individuelle Unterschiede gibt. Leider geht die Schere in der Sprachkompetenz im Verlauf der Entwicklung immer weiter auseinander. "Wortarmut“ (Moats in Wolf M. 2010, 122) aufgrund mangelnder familiärer Kommunikation und nicht vorhandenen Lesestoffs wirkt sich verheerend auf die weitere Sprachentwicklung und das Lesen aus.

Weitere interessante Einblicke zur Sprachentwicklung bietet das Video von Mayberry (2015). Im Laufe der Sprachentwicklung verändert sich das Gehirn. Apropos „verändern“: Wie wird sich das sprachliche Vermögen unserer Kinder und Kindeskinder weiterentwickeln, wie wird sich das Gehirn angesichts der elektronischen Medien verändern? 


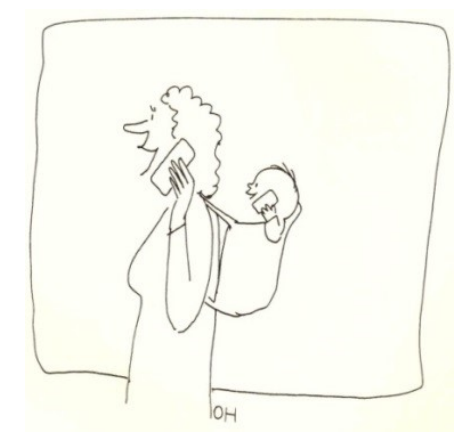

Abbildung 10: Gehirn\&Geist 2011, 7-8, 83

Die Evolution hat uns den aufrechten Gang, durch Veränderung des Gehirns das Denken und eben auch die Sprache gebracht. Umwelt- und Lerneinflüsse wirken auch heute noch auf das Gehirn ein. Eine spannende Frage: Wie verändert die veränderte Kommunikation und auch das veränderte Lesen (Simsen, Chatten, Surfen, E-mailen) das Hirn? Führen diese neuen Medien zur „digitalen Demenz“, wie Spitzer (2012) meint? Hier ist sicher noch viel Forschung notwendig, um die Positiva und die Negativa der ,digitalen Welt ${ }^{\star}$ klar zu formulieren!

Einen kurzen Überblick über das, was sich alles im Gehirn durch das Lernen von Sprachen verändert, gibt das Video „How Does Language Change Your Brain?“: https:// www.youtube.com/watch?v = aGITqiG-Ips; veröffentlicht am 11.08.2015.

\section{Musik und Sprache}

Nach Levitin ist der „Musik-Instinkt der Hit der Evolution“ (2009, 317). Die Fähigkeit, sich mit Kunst (in ,Bild- und Musiksprache') auszudrücken, ist möglicherweise der gesprochenen Sprache vorausgegangen. Manche meinen, es war umgekehrt. Wie auch immer die Reihenfolge gewesen sein mag, Musik und Sprache sind,verwandt'. Es gibt Ähnlichkeiten hinsichtlich der akustischen Parameter (Frequenz, Dauer, Intensität, Klangfarbe). Beide, sowohl Musik als auch Sprache, implizieren Aufmerksamkeit, Gedächtnis, Rhythmus, Melodie, Harmonie, Emotionen, und auch Bedeutungen. Musik und Sprache nützen teilweise „overlapping neural resources“ (Zioga, I., Di Bernardi Luft C., Bhattacharya, J. 2016, 267): Die Tonlage ist sowohl für die Melodie in der Musik als auch für die Prosodie (Betonung, rhythmische Gliederung) bei der Sprache wichtig. Die Rhythmen, sowohl in der Musik als auch in der Sprache, haben eine Entsprechung in sogenannten Oszillationen (elektrischen Schwingungen) im Gehirn: Es gibt solche Schwingungen, die den Tempi in der Musik entsprechen, und auch Rhythmen, die mit Sprache synchronisiert sind (Max-Planck-Gesellschaft 2015b). Ein wesentliches gemeinsames Feld für Sprache und Musik ist das Broca-Areal im Schläfenlappen: Es ist nicht 
nur für die Sprachproduktion zuständig, sondern „speziell dafür, verschiedene Elemente zu einem Gesamtbild zusammenzufügen. [... In der Sprachverarbeitung müssen Einzelwörter zu Sätzen kombiniert werden, im Fall von Musik einzelne Töne zu Melodien“ (Max-Planck-Gesellschaft 2015a; siehe auch Kuhnke 2017).

Levitin (2009) entdeckte, dass Musikhören Teile der Sprachregionen in der linken Hälfte aktivierte, interessanterweise aber auch eine analoge Region in der rechten Hemisphäre (156-157). Und auch das Kleinhirn, das bei der Sprache eine wichtige Rolle spielt (siehe oben), ist in Musik involviert: Beim Anhören von Musik wurden starke Aktivierungen in diesem Hirnteil nachgewiesen (Levitin 2009, 217).

Musik und Sprache beeinflussen sich positiv. So finden sich bei musikalischer Erfahrung z. B. eine verbesserte Tonhöhenverarbeitung in der Musik und in der Sprache sowie eine verbesserte phonetische Kategorisierung, mit höherer Aktivierung des linken Planum temporale, bei Musikern als bei Nichtmusikern. Musikalische Expertise verbesserte sowohl die Wahrnehmung linguistischer Merkmale in der Fremdsprache als auch die Wahrnehmung der Zeitstruktur in der Mutter- und Fremdsprache. Höhere musikalische Fähigkeiten korrelierten mit einer guten „phonetic coding ability“ und einer besseren Aussprache in der Fremdsprache (Turker 2017). Musikalisch kreative Menschen haben größere Hirnoberflächen und Volumina in den relevanten Arealen für die beteiligte Motorik, die Sound-Verarbeitung, die Sprache, im sogenannten „Ruhe-Netzwerk“ und für die Emotionen (Bashwiner et al. 2016). Dass Musik und Sprache teilweise dieselben Hirnareale (z. B. den rechten Auditorischen Cortex) verwenden, zeigt sich schon bei Babys (Perani et al., 2011). Für Babys ist die Melodielinie die hervorstechendste Eigenschaft. Das passt auch gut zur Sprachentwicklung (siehe auch die ,Melodie“ des Babytalk). 


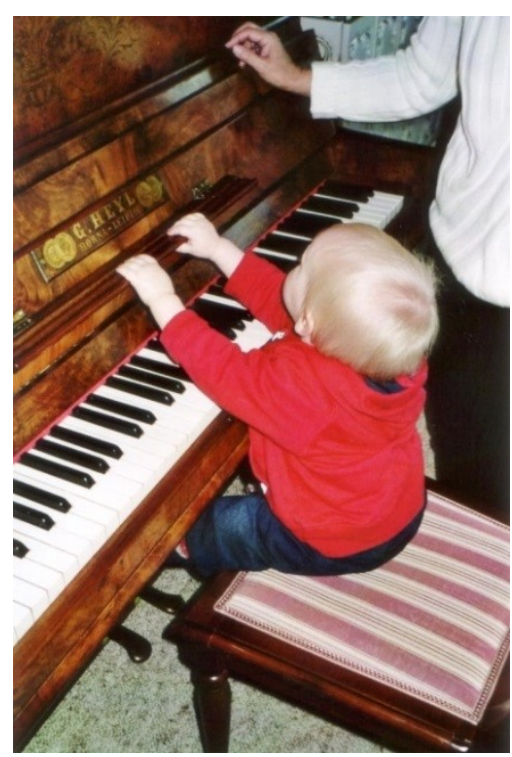

Abbildung 11: Foto: Privat

Das Erlernen eines Instruments in der Kindheit trainiert das Gehirn und hat damit auch positive Auswirkungen auf die späteren kognitiven Leistungen (Beras 2014; Dalla Bella und Tillmann 2015). Auch das Hören von Musik (Altenmüller et al. 2014; Rollnik/Altenmüller 2014; Zatore et al. in Suzuki und Fitzpatrick 2016, 54) aktiviert viele Hirnareale in den Frontal- und Schläfenlappen (auch links), sogar im Kleinhirn und natürlich vor allem im Limbischen System (Striatum, Amygdala, Hippocampus). Dort wirkt es sich auf das Belohnungssystem (Nucleus accumbens, Ventral tegmentales Areal) aus. Es führt nicht nur zu emotionalem Wohlbefinden, sondern beeinflusst kognitive Prozesse, damit auch die Sprache positiv. Das wird auch dadurch erreicht, dass der Blutdurchfluss im Limbischen System verbessert wird.

\section{Ein kurzes Schlusswort}

„Je mehr wir über das Gehirn lernen, desto mehr Wege werden wir finden, seine Funktion zu verbessern.“ (Minsky 1995, 83).

Möge uns die Hirnforschung - interdisziplinär mit Psychologie, Linguistik, usw. viele wertvolle Erkenntnisse liefern für das ,gnoti sauton', das ,Erkenne Dich selbst', damit wir besser lehren und lernen, lesen und damit hoffentlich auch die vielen Probleme und Herausforderungen besser meistern können! Bei all diesen Bestrebungen wissen wir 
natürlich, dass unsere Erkenntnisse immer nur vorläufige sind, „Annäherungen an die Wahrheit“" (Popper 1994, 39).

Und für das schulische Lernen insgesamt dürfen wir nicht vergessen:

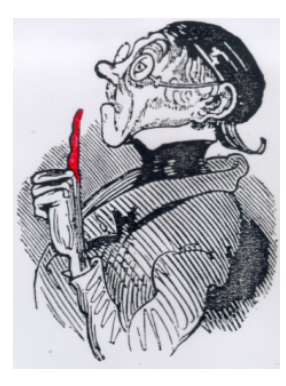

Trotz Tests und Prüfungen aller Art ist die Bildungsphilosophie alter Pädagogen aktueller denn je: „Nicht allein das ABC bringt den Menschen in die Höh', nicht allein in Rechnungssachen soll der Mensch sich Mühe machen, ... sondern auch der Weisheit Lehren muss man mit Vergnügen hören“ (Busch 2005, 1). ,Bildung' muss ,umfassend" sein, nicht nur Ausbildung und nicht nur an Messungen orientiert! Diese Forderung nach Ganzheitlichkeit ist hirnbiologisch sehr gut begründbar!

\section{Literatur}

Aamodt, S., and S. Wang. 2012. Welcome to Your Child's Brain. Die Entwicklung des kindlichen Gehirns von der Zengung bis zum Reifezengnis. München: Beck.

Altenmüller, E., Siggel, S., Mohammadi, B., Samii, A., Münte, T . F. 2014. „Play it Again, Sam: Brain Correlates of Emotional Music Recognition. “Frontiers in Psychology, Feb. 18. doi: 10.3389 /fpsyg.2014.00114

Apple, D. M. Fonseca, R. S, Kokovay, E. 2017. „The Role of Adult Neurogenesis in Psychiatric and Cognitive Disorders." Brain Research 1655: 270-276.

Bardin, J. 2013. Alles zu seiner Zeit. GehirnEGeist, 1-2, 52-57.

Bareither, I. 2014. Eine Bildergeschichte des Gehirns. GehirnEGeist, 5, 40-47.

Bashwiner, D. M., Wertz, C. J., Flores, R. A., Jung, R. E. 2016. „Musical Creativity ,Revealed“ in Brain Structure: Interplay between Motor, Default Mode, and Limbic Networks." Scientific Reports 6, doi: 10.1038/srep20482.

Beras, E. 2014. „Young Musicians Reap Long-Term Neuro Benefits.“ Scientific American, Feb 22. https://www.scientificamerican.com/podcast/episode/young-musicians-reap-long-term-neurobenefits/.

Berezutskaya, J., Freudenburg, Z. V., Güçlü, U., van Gerven, M. A. J., Ramsey, N. F. 2017. „Neural Tuning to Low-Level Features of Speech throughout the Perisylvian Cortex.“ Journal of Neuroscience 37 (33): 7906-7920.

Berger, S. o. J. „Wenn die Wörter laufen lernen.“ In GehirnEGeist Serie, 2., überarbeitete Auflage. Heidelberg: Spektrum der Wissenschaft. 
Bergmann, O. und H. Huttner. 2015. „Aus der Trickkiste der Neuroarchäologen.“ GehirnEGeist 10: $54-58$.

Berk, L. E. 2005. Entwicklungspsychologie, 3., aktualisierte Auflage. München: Pearson Education. Berninger, B. und M. Götz. 2009. „Nachwuchsförderung im Gehirn.“ GehirnEGeist 7-8: 58-63.

Blakemore, S.-J. und U. Frith. 2006. Wie wir lernen. Was die Hirnforschung darüber weiß. München: Deutsche Verlags-Anstalt.

Busch, W. 2005. „Max und Moritz. Vierter Streich.“, May 28, 2018. http://www.wilhelm-buschseiten.de/werke/maxundmoritz/streich4.html

CARTA: How Language Evolves: Language in the Brain. Public Symposium, Feb. 20, 2015. https://www.youtube.com/watch?v=0aFMzANQd7A.

Chang, E. 2015. „Feature Taxonomy in Speech Auditory Cortex.“ CARTA: How Language Evolves: Language in The Brain. Public Symposium, Feb. 20, 2015. https://www.youtube.com/wat ch?v=0aFMzANQd7A.

Cook, G. 2010. „Das Gehirn und das geschriebene Wort.“ GehirnEGeist 10: 22-25.

Dalla Bella, S. und B. Tillmann. 2015. „Der Rhythmus des Gehens.“ GehirnEGeist 1: 44-49.

Danielson, N. 2016. „Newborn Neurons Keep Memories Crisp and Fresh.“ Redigiert von Emily Underwood in http://www.sciencemag.org/news/2016/03/newborn-neurons-keep-memoriescrisp-and-fresh. DOI: $10.1126 /$ science.aaf4174.

Dehaene, S. 2008. Neuroscience. Presentation for Dyslexia International's Online conference, November 2008. http://www.dyslexia-international.org/neuroscience.

Dehaene, S. und L. Cohen. 2011. „The Unique Role of the Visual Word Form Area in Reading.“ Trends in Cognitive Sciences 15 (6): 254-262.

Dehaene, S. 2013. „Reading the Brain.“, April 30. https://www.youtube.com/watch?v=MSy $685 \mathrm{vNqYk}$

Ding, N., Melloni, L., Zhang, H., Tian, X., Poeppel, D. 2016. „Cortical Tracking of Hierarchical Linguistic Structures in Connected Speech.“ Nature Neuroscience 19: 158-164.

Elman, J. 2008. „Language and the Human Brain.“, Jan 22, https://www.youtube.com/watch?v= K1pbnWcabMY

Fedorenko, E. 2015. „Specialization for Language in the Human Brain.“ CARTA: How Language Evolves: Language in The Brain. Public Symposium, Feb. 20, 2015. https://www.youtube.com/ watch? $\mathrm{v}=0 \mathrm{aFMzANQd7A.}$

Förstl, H., M. Hautzinger, G. Roth. 2006. Neurobiologie psychischer Störungen. Heidelberg: Springer Medizin Verlag.

Gaarder, J. 1996. Sofies Welt. Roman über die Geschichte der Philosophie. München: Hanser.

Gehirn\&Geist SPEZIAL. 2011. „Entdeckungsreise durch das Gehirn. Die Sprachzentren.“ 1: 6669.

Gehirn\&Geist. 2011. Abbildung. 7-8, 83.

Giraud, A.-L. 2017. „Neural Oscillations in Speech and Language.“ Symposium in Berlin. https:// www.youtube.com/watch?v=kJUOTYIZhUk

Goethe, J. W. v. 1808. Faust I. Tübingen: Cotta. Faksimileausgabe des Erstdruckes von 1808. Frankfurt am Main: Insel Verlag.

Grey, S. et al. 2017. „Bilingual and Monolingual Adults Learning an Additional Language: ERPs Reveal Differences in Syntactic Processing. “ Oct 2, https://doi.org/10.1017/S1366728917000426.

Grisoni, L., T. Miller, F. Pulvermüller. 2017. „Neural Correlates of Semantic Prediction and Resolution in Sentence Processing.“ Journal of Neuroscience 37 (18): 4848-4858. 
Heer, W. de, Huth, A. G., Griffiths, T. L., Gallant, J. L., Frédéric E. Theunissen, F. E. 2017. „The Hierarchical Cortical Organization of Human Speech Processing." Journal of Neuroscience 37 (27): 6539-6557.

Hobel, B. 2017. „Was ist Unterstützte Kommunikation? Sprachwissenschaft in der Praxis.“ Vortrag beim Workshop „Sprache und Gehirn“ der Österreichischen Linguistiktagung in Klagenfurt, Dec. 10.

Kauschke, C., C. Nutsch, J. Schrauf. 2012. „Verarbeitung von konkreten und abstrakten Wörtern bei Kindern im Schulalter.“ Zeitschrift für Entwicklungspsychologie und Pädagogische Psychologie 44 (1): 2-11.

Kempermann, G. 2006. „Neurogenese. Kopfgeburten.“ GehirnEGeist 3: $28-32$.

Kempermann, G. 2017. „Das Gedächtnis updaten.“ GehirnEGeist 1: 52-55.

Kheirbek, M., R. Hen. 2015. „Zu viel Gespür für Gefahr.“ GehirnEGeist 10: 48-53.

Kim, J. S., Kanjlia, S., Merabet L. B., Bedny, M. 2017. „Development of the Visual Word Form Area Requires Visual Experience: Evidence from Blind Braille Readers. "JNeurosci. The Journal of Neuroscience. https://doi.org/10.1523/JNEUROSCI.0997-17.2017.

Kocagoncu, E., Clarke, A., Devereux, B. J., Tyler, L. K. 2017. „Decoding the Cortical Dynamics of Sound-Meaning Mapping." Journal of Neuroscience 37 (5): 1312-1319.

Kuhl, P. 2011. „The Linguistic Genius of Babies.“, Feb 18, https://www.youtube.com/watch?v= G2XBIkHW954

Kuhnke, P. 2017. „Transcranial Magnetic Stimulation (TMS) in the Neuroscience of Language.“ Vortrag beim Workshop „Sprache und Gehirn“ der Österreichischen Linguistiktagung in Klagenfurt, Dec. 10.

Kumar, V., Pandey, A., Jahan, J., Kumar Shukla, R., Kumar, D.,Srivastava, A., Singh, S., Rajpurohit, C. S., Yadav, S., Kumar Khanna, V., Pant, A. B. 2016. „Differential Responses of Trans-Reservatrol on Proliferation of Neural Progenitor Cells and Aged Rat Hippocampal Neurogenesis. “ Scientific Reports 6: 28142.

Lesage, E., Hansen, P. C., Miall, R. C. 2017. „Right Lateral Cerebellum Represents Linguistic Predictability." Journal of Neuroscience 37 (26): 6231-6241.

Levitin, D. J. 2009. Der Musik-Instinkt. Die Wissenschaft einer menschlichen Leidenschaft. Heidelberg: Spektrum.

Ma, D. K., Dengke, K., MaMi-Hyeon, J., Junjie, U., Guo Hongjun, S. 2009. „Neuronal ActivityInduced Gadd45b Promotes Epigenetic DNA Demethylation and Adult Neurogenesis. "Science, Feb 20, 1074-1077.

Max-Planck-Gesellschaft. 2015a. „Doppelte Herausforderung fürs Gehirn. Sprache und Musik werden in der gleichen Hirnregion verwaltet.“ http://www.mpg.de.

Max-Planck-Gesellschaft. 2015b. „Gehirn im Takt mit der Musik.“ http://www.mpg.de.

Max-Planck-Institut für empirische Ästhetik. 2017. „Neural Oscillations in Auditory Cognition, Music, Speech, and Language.“ https://www.aesthetics.mpg.de/forschung/abteilung-neurowi ssenschaften/neural-oscillations.html.

Mayberry, R. 2015. „How the Environment Shapes the Language in the Brain.“ CARTA: How Language Evolves: Language in the Brain. Public Symposium, Feb 20, https://www.youtube. com/watch?v=0aFMzANQd7A.

Minsky, M. 1995. „Werden Roboter die Erde beherrschen?“ Spektrum der Wissenschaft Spezial, Leben im Kosmos, 80-86. 
Perani, D., Saccuman, M. C., Scifo, P., Anwander, A., Spada, D., Baldoli, C., Poloniato, A., Lohmann, G., Friederici, A. D. 2011. „Neural Language Networks at Birth.“ Proceedings of the National Academy of Sciences of the United States of America 108 (38): 16056-16061.

Popper, K. R. 1994. Alles Leben ist Problemlösen. Über Erkenntnis, Geschichte und Politik. München: Piper.

Reiter, M. 2014. „Sag’s einfach!“ GehirnEGeist 1-2: 62-67.

Rollnik, J. D. und E. Altenmüller. 2014. „Music in Disorders of Consciousness.“ Frontiers in Neuroscience, Jul. 3.

Roth, G. 2011. Bildung braucht Persönlichkeit. Wie Lernen gelingt. Stuttgart: Klett-Cotta.

Roth, G. und N. Strüber. 2015. Wie das Gehirn die Seele macht. Stuttgart: Klett-Cotta.

Saygin, Z. M., Osher, D. E., Norton, E. S., Youssoufian, D. A., Beach, S. D., Feather, J., Gaab, N., Gabrieli, J. D. E., Kanwisher, N. 2016. „Connectivity Precedes Function in the Development of the Visual Word Form Area." Nature Neuroscience 19: 1250-1255.

Schachl, H. 1980. Die Abbängigkeit des Sprachverstehens bei der Tachistophonen Methode von Lernen, Intelligenz und Gedächtnisprozessen. Dissertation an der Naturwissenschaftlichen Fakultät der Universität Salzburg.

Schachl, H. 2012. Was haben wir im Kopf? Die Grundlagen für gebirngerechtes Lebren und Lernen. 3. aktualisierte und überarbeitete Auflage. Linz: Veritas.

Schlaggar, B. L. und B. D. McCandliss. 2007. „Development of Neural Systems for Reading.“ Annual Review of Neuroscience 30: 475-503.

Schneider, W. und U. Lindenberger. 2012. Entwicklungspsychologie. 7., vollständig überarbeitete Auflage. Weinheim: Beltz.

Schoenfeld, T. J. und H. A. Cameron. 2015. „Adult Neurogenesis and Mental Illness.“ Neuropsychopharmacology Reviews 40: 113-128.

Shors, T. J. 2009. „Saving New Brain Cells.“ Scientific American 3: 41-48.

Skaggs, W. 2015. „Nachwuchs fürs Gedächtnis.“ GehirnEGeist 7: 60-65.

Spitzer, M. 2012. Digitale Demenz: Wie wir uns und unsere Kinder um den Verstand bringen. München: Droemer HC.

Stiles, J. 2008. „Brain Development.“ Jan 22, http://www.youtube.com/watch?v=FugrcVhi2tg

Suzuki, W. und B. Fitzpatrick. 2016. Fittes Gehirn, Erfülltes Leben. München: Goldmann. (Englische Version: (2015): Healthy Brain, Happy Life. Verlag Dey Street.

Turker, S. 2017. „The Neuroanatomy of Foreign Language Aptitude.“ Vortrag beim Workshop „Sprache und Gehirn“ der Österreichischen Linguistiktagung in Klagenfurt, Dec. 10.

Wolf, C. 2010. „Lob des Lesens.“ GehirnEGeist 10: 14-20.

Wolf, M. 2010. Das lesende Gehirn. Wie der Mensch zum Lesen kam - und was es in unseren Köpfen bewirkt. Heidelberg: Spektrum Akademischer Verlag.

Wolf, M., M. Barzillai, E. Norton. 2008. „Routes to Reading.“ In The Reading Brain, March 25, http://science-community.sciam.com/blog-entry/Mind-Matters/Reading-Brain/580000

Yao, B. et al. 2016. „Epigenetic Mechanism in Neurogenesis.“ Nature Reviews Neuroscience, June 23, doi: $10.1038 / \mathrm{nrn} .2016 .70$

Zioga, I., Di Bernardi Luft C., Bhattacharya, J. 2016. „Musical Training Shapes Neural Responses to Melodic and Prosodic Expectation.“ Brain Research 1650: 267-282. 